\title{
ROBUST STABILITY ANALYSIS FOR STRUCTURED UNCERTAINTIES WITH BOUNDED VARIATION RATES
}

\author{
Hakan Köroğlu Carsten W. Scherer \\ Delft Center for Systems and Control, Delft University of Technology \\ Mekelweg 22628 CD Delft The Netherlands \\ Phone: +31(0)1527\{87171,85899\} Fax : +31(0)152786679 \\ E-Mail : $\{$ h.koroglu,c.w.scherer\}@dcsc.tudelft.nl
}

\begin{abstract}
Robust stability analysis problem is considered for the feedback interconnection of a causal and stable linear time-invariant system with causal and stable linear time-varying block-diagonal structured uncertainties having subblocks of bounded norms and variation rates. The problem is studied within the recently developed IQC framework of robust stability analysis, and based on a generalized version of the swapping lemma, a multiplier class capturing the considered uncertainties is obtained and thus a robust stability test is developed in terms of an LMI feasibility problem. Copyright (c)2005 IFAC
\end{abstract}

Keywords: Robust stability, stability analysis, structured uncertainty, slowly time-varying systems, bounded-rate parameter variations, linear matrix inequalities (LMI), integral quadratic constraints (IQC).

\section{INTRODUCTION}

There has been significant amount of research work viewing the controller design problem as a design that is to be performed for a nominal system, which might be affected by some perturbations (uncertainties), and the wide domain of these efforts is by now commonly referred to as robust control, with the robust stability analysis constituting a major part. In this paper, we consider the robust stability analysis problem for structured linear time-varying (LTV) uncertainties with bounded norms and rates-of-variation. Our interest in this problem is intrigued mainly by the results of Poolla and Tikku [1995]; Jönsson and Rantzer [1996], and we are especially interested in developing nonconservative and numerically efficient robust stability analysis tests. For -at least a partial- achievement of this goal, we employ the IQC approach of Megretski and Rantzer [1997] to robust stability analysis and make use of LMI methods. We start with some preliminaries, followed by the description of the considered problem and the relevant literature. Section 4 summarizes the IQC approach to robust stability analysis and Section 5 presents our contributions. We also provide an example, after which we conclude by some final remarks.

\section{NOTATION AND PRELIMINARIES}

We work in a discrete-time setting with squaresummable sequences, $x \in \ell_{2}^{n}$, as signals and linear operator maps as systems (see e.g. Willems [1971] for some preliminaries). We treat real-valued matrices, $X \in \mathbb{R}^{m \times n}$; real-rational transfer functions, $H \in \mathcal{R} \mathcal{L}_{\infty}^{m \times n}$; and bounded linear maps, $T \in \mathcal{L}^{m \times n}$, from $\ell_{2}^{n}$ into $\ell_{2}^{m}$, in a unified setting all as operators. In particular, we refer to the set of symmetric positive-definite matrices by $\mathbb{P}^{m \times m}$; to the set of stable and causal transfer functions by $\mathcal{R H}_{\infty}^{m \times n}$; and to the set of causal and unity norm-bounded linear operators by $\mathcal{B L}^{m \times n} \triangleq\{T \in$ $\left.\mathcal{L}^{m \times n}:\|T\|_{\text {i2 }} \leq 1\right\}$, where $\|\cdot\|_{\text {i2 }}$ denotes the induced $\ell_{2}$-norm. We denote the identity operator of size $m$ by $I_{m}$; right shift (delay) operator of size $m$ by $Z_{m}^{-1} \triangleq z^{-1} \otimes I_{m} ;$ and the zero operator of size $m \times n$ by $0_{m \times n}$. For a given linear operator $\Delta \in \mathcal{L}^{m \times n}$, we define its variation as $\mathcal{V}_{\Delta} \triangleq \Delta Z_{n}^{-1}-Z_{m}^{-1} \Delta$ and refer to $\left\|\mathcal{V}_{\Delta}\right\|_{\mathrm{i} 2}\left(\leq 2\|\Delta\|_{\mathrm{i} 2}\right)$ as the rate-ofvariation of $\Delta$. For a compact representation of structures, we denote the block-diagonal operators as $\left[T_{i}\right]_{i=1}^{\kappa} \triangleq \operatorname{diag}\left[T_{1}, \ldots, T_{\kappa}\right]$ and employ the Kronecker product to describe the commuting structures. We make use of the following Kronecker product commutation identity, which will generalize as the swapping lemma in the sequel: 
Lemma 1. (Kronecker Product Commutation Identity) Let $T_{1} \in \mathbb{R}^{m_{1} \times n_{1}} \& T_{2} \in \mathcal{L}^{m_{2} \times n_{2}}$. Then we have

$$
\left(I_{m_{2}} \otimes T_{1}\right)\left(T_{2} \otimes I_{n_{1}}\right)=\left(T_{2} \otimes I_{m_{1}}\right)\left(I_{n_{2}} \otimes T_{1}\right) .
$$

The identity is valid also for the case $T_{1} \in$ $\mathcal{R} \mathcal{H}_{\infty}^{m_{1} \times n_{1}}$ and $T_{2} \in \mathcal{R} \mathcal{H}_{\infty}^{m_{2} \times n_{2}}$.

We work mainly in an IQC-based robust stability analysis setting, which is established on some basic notions in inner product spaces (see e.g. Jönsson [2001]). With the inner product of $x, y \in \ell_{2}^{n}$ defined as $\langle x, y\rangle \triangleq \sum_{t} x^{T}(t) y(t)$, the adjoint of an operator $T \in \mathcal{L}^{m \times n}$ is defined as the unique operator, $T^{*} \in \mathcal{L}^{n \times m}$, for which $\langle T x, y\rangle=\left\langle x, T^{*} y\right\rangle, \forall x \in \ell_{2}^{n}, \forall y \in \ell_{2}^{m}$. A selfadjoint operator, $T=T^{*} \in \mathcal{L}^{m \times m}$, is described as positive semi-definite $(T \geq 0)$ if it satisfies $\langle T x, x\rangle \geq 0, \forall x \in \ell_{2}^{m}$. Within the environment defined by these notions, we employ in the sequel the following lemma, which is a slightly modified version of a standard inequality in operator theory:

Lemma 2. (Bounding Lemma) Let $T_{1}, T_{2} \in \mathcal{L}^{m \times n}$ and $X \in \mathbb{P}^{m \times m}$. Then we have

$$
\mp\left(T_{1}^{*} T_{2}+T_{2}^{*} T_{1}\right) \leq T_{1}^{*} X T_{1}+T_{2}^{*} X^{-1} T_{2} .
$$

\section{PROBLEM FORMULATION}

The standard setup of stability analysis is given in Figure 1, where $M \in \mathcal{R} \mathcal{H}_{\infty}^{\eta \times \eta}$ denotes a known causal and stable LTI operator (which is referred to as the plant) and $\Delta \in \mathcal{L}^{\eta \times \eta}$ denotes some causal LTV operator (which is referred to as the uncertainty or the perturbation). The feedback interconnection in Figure 1 is well-posed (or in short $(M, \Delta)$ is well-posed), if, for any $u_{1}, u_{2} \in$ $\ell_{2 \mathrm{e}}^{\eta}$, there exist $e_{1}, e_{2} \in \ell_{2 \mathrm{e}}^{\eta}$, which depend causally on $u_{1}, u_{2} \in \ell_{2 \mathrm{e}}^{\eta}$ (see Willems [1971] for a detailed discussion of well-posedness). We say that the system in Figure 1 is $\ell_{2}$-stable, if we have $e_{1}, e_{2} \in$ $\ell_{2}^{\eta}$ whenever $u_{1}, u_{2} \in \ell_{2}^{\eta}$. We say that $M$ is robustly stable against $\Delta$, if the feedback interconnection of $M$ and $\Delta$ is stable for all $\Delta \in$ $\boldsymbol{\Delta}$, where $\boldsymbol{\Delta}$ denotes the set of uncertainties. In this setting, we consider the following problem:

Problem 3. Consider the feedback system in Figure 1 with a given causal and stable LTI plant $M \in \mathcal{R} \mathcal{H}_{\infty}^{\eta \times \eta}$, together with causal and stable structured LTV uncertainties $\Delta$ that come out of the set $\boldsymbol{\Delta}=\boldsymbol{\Delta}_{\nu}^{s} \subset \mathcal{B} \mathcal{L}^{\eta \times \eta}$, which is defined as

$$
\boldsymbol{\Delta}_{\nu}^{s} \triangleq\left\{\begin{array}{l}
{\left[I_{l_{i}} \otimes \Delta_{i}\right]_{i=1}^{\kappa}: \Delta_{i} \in \mathcal{B L}^{m_{i} \times m_{i}},} \\
\left\|\mathcal{V}_{\Delta_{i}}\right\|_{\mathrm{i} 2} \leq \nu_{i} ; i=1: \kappa, \Delta_{i}^{*}=\Delta_{i} ; i=1: \kappa_{1} \leq \kappa
\end{array}\right\},
$$

where $s=\left\{l_{i}, m_{i} \times m_{i}\right\}_{i=1}^{\kappa} ; \eta=\sum_{i=1}^{\kappa} l_{i} m_{i}$ and $\nu=\left\{\nu_{i}\right\}_{i=1}^{\kappa} ; \nu_{i} \in[0,2]$ describe, respectively, the structure and the maximum rate-of-variation of the uncertainty, and $\kappa_{1}$ denotes the number of selfadjoint channels (considered basically with motivations coming from parametric uncertainties). Determine if $M$ is robustly stable against $\boldsymbol{\Delta}_{\nu}^{s}$.

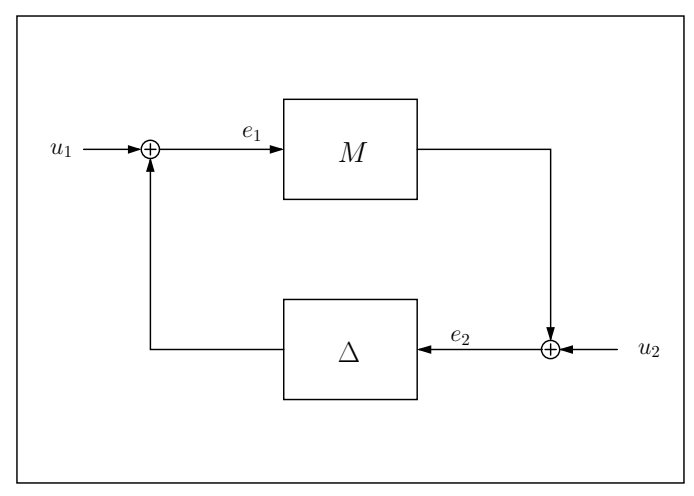

Fig. 1.Standard setup for robust stability analysis.

There are various results available in the literature as partial solutions to Problem 3. It is wellestablished by now that the structured singular value analysis provides an exact test (under some minor technical assumptions) for robust stability analysis in the linear time-invariant (LTI) perturbations case, i.e. $\boldsymbol{\Delta}=\boldsymbol{\Delta}_{0}^{s}$, which is of natural interest as a limiting case within the description of Problem 3. Due to the computational complexity of the structured singular value calculation, analysis is based on upper bound calculation using Dscales, and $\exists H, H^{-1} \in \mathcal{R} \mathcal{H}_{\infty}^{\eta \times \eta}$ s.t. $H \Delta=\Delta H, \forall \Delta \in$ $\Delta \&\left\|H M H^{-1}\right\|_{\infty}<1$, is provided as a sufficient test for robust stability (see Packard and Doyle [1993] for details/extensions). Although D scaling test is known to be conservative for certain LTI perturbations, it has been shown by Shamma [1994] in $\ell_{2}$ framework that the test with constant D-scales, $H \in \mathbb{R}^{\eta \times \eta}$, is exact (under some technical assumptions) in the case of arbitrarily timevarying block diagonal uncertainties with full subblocks, i.e. $\boldsymbol{\Delta}=\boldsymbol{\Delta}_{2}^{s}$ (see also Paganini and Doyle [1994];Meinsma et al. [2000] for some generalizations). The case of slowly time-varying perturbations was considered by Poolla and Tikku [1995] for uncertainties with full sub-blocks, the ratesof-variation of which are all bounded by the same value and it was shown that frequency-dependent D-scales test is necessary and sufficient in the case of strictly proper plants perturbed with arbitrarily slowly time-varying uncertainties. In particular, a conservative bound of the maximum allowable rate-of-variation was provided in terms of the Dscales. Yet, let alone exactness, an efficient calculation of this bound by some sort of search over D-scales seems to be difficult if at all possible. The problem was considered with concerns about numerical efficiency by Jönsson and Rantzer [1994, 1996] for scalar repeated uncertainties and an IQC-based robust stability test was developed in a continuous-time setting. A slight improvement to this was reported by Helmersson [1999]. There are also some relevant works by Tchernychev and Sideris [1999, 2000], who also study the problem for repeated scalar uncertainties with a slightly different approach. 


\section{IQC-BASED ROBUST STABILITYANALYSIS}

Among the various others, the IQC approach has recently emerged as a unifying framework of robust stability analysis based on operator methods. We summarize the central result by Megretski and Rantzer [1997] in the following theorem, with adaptations from Jönsson [2001]:

Theorem 4. (IQC Stability, Megretski and Rantzer [1997]) Let the set $\boldsymbol{\Delta}_{\Pi}$ be defined as

$$
\boldsymbol{\Delta}_{\Pi} \triangleq\left\{\Delta \in \mathcal{L}^{\eta \times \eta}:\left[\begin{array}{c}
I_{\eta} \\
\Delta
\end{array}\right]^{*}\left[\begin{array}{ll}
\Pi_{11} & \Pi_{12} \\
\Pi_{12}^{*} & \Pi_{22}
\end{array}\right]\left[\begin{array}{c}
I_{\eta} \\
\Delta
\end{array}\right] \geq 0\right\}
$$

where the IQC multiplier $\Pi \in \mathcal{R} \mathcal{L}_{\infty}^{2 \eta \times 2 \eta}$ is a selfadjoint transfer function formed by the sub-blocks $\Pi_{11}=\Pi_{11}^{*}, \Pi_{22}=\Pi_{22}^{*}, \Pi_{12} \in \mathcal{R} \mathcal{L}_{\infty}^{\eta \times \eta}$. The feedback system of Figure 1 is robustly stable against $\Delta$ if the following conditions are all satisfied:

(i) $(M, \tau \Delta)$ is well-posed $\forall \tau \in[0,1]$ and $\forall \Delta \in \Delta$.

(ii) $\tau \Delta \in \boldsymbol{\Delta}_{\Pi}, \forall \tau \in[0,1]$ and $\forall \Delta \in \boldsymbol{\Delta}$.

(iii) There exists an $\epsilon \in \mathbb{R}_{+}$such that, $\forall \omega \in[-\pi, \pi]$,

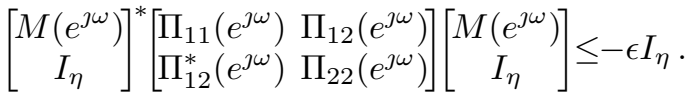

PROOF. See Megretski and Rantzer [1997].

Remark 5. The well-posedness of the feedback loop will be assured if $M$ is strictly proper. If the IQC multiplier satisfies $\Pi_{11} \geq 0 \& \Pi_{12}=0$ or $\Pi_{11} \geq 0 \& \Pi_{22} \leq 0$, we have $\tau \Delta \in \boldsymbol{\Delta}_{\Pi}$ for $\tau \in[0,1]$, whenever $\Delta \in \boldsymbol{\Delta}_{\Pi}$.

After an appropriate factorization of the multiplier, the frequency domain inequality in (5) can be transformed into an LMI by employing the (discrete-time and strict version of the) celebrated Kalman-Yakubovich-Popov (KYP) lemma:

Lemma 6. (Kalman-Yakubovich-Popov Lemma) Let $\Phi \in \mathcal{R} \mathcal{H}_{\infty}^{m_{\Phi} \times n_{\Phi}}$ be a causal and stable transfer function that has a realization of the form $\Phi=\left[\begin{array}{c|c}A_{\Phi} & B_{\Phi} \\ \hline C_{\Phi} & D_{\Phi}\end{array}\right] \triangleq C_{\Phi}\left(Z_{k_{\Phi}}-A_{\Phi}\right)^{-1} B_{\Phi}+D_{\Phi}$, where $A_{\Phi} \in \mathbb{R}^{k_{\Phi} \times k_{\Phi}}, B_{\Phi} \in \mathbb{R}^{k_{\Phi} \times n_{\Phi}}, C_{\Phi} \in \mathbb{R}^{m_{\Phi} \times k_{\Phi}}, D_{\Phi} \in$ $\mathbb{R}^{m_{\Phi} \times n_{\Phi}}$, with $A_{\Phi}$ having all its eigenvalues strictly inside the unit disk, and let $Q=Q^{T} \in$ $\mathbb{R}^{m_{\Phi} \times m_{\Phi}}$ be a symmetric matrix. Then the following conditions are equivalent:

(i) There exists an $\epsilon \in \mathbb{R}_{+}$such that

$$
\Phi^{*}\left(e^{\jmath \omega}\right) Q \Phi\left(e^{\jmath \omega}\right) \leq-\epsilon I_{n_{\Phi}}, \forall \omega \in[-\pi, \pi] .
$$

(ii) There exists a symmetric matrix $P=P^{T} \in$ $\mathbb{R}^{k_{\Phi} \times k_{\Phi}}$ and an $\epsilon \in \mathbb{R}_{+}$such that

$\left[\begin{array}{ll}I_{k_{\Phi}} & 0 \\ A_{\Phi} & B_{\Phi} \\ C_{\Phi} & D_{\Phi}\end{array}\right]^{T}\left[\begin{array}{rrr}-P & 0 & 0 \\ 0 & P & 0 \\ 0 & 0 & Q\end{array}\right]\left[\begin{array}{ll}I_{k_{\Phi}} & 0 \\ A_{\Phi} & B_{\Phi} \\ C_{\Phi} & D_{\Phi}\end{array}\right] \leq-\epsilon I_{n_{\Phi}}$.

PROOF. See e.g. Rantzer [1996].

\section{AN IQC-BASED ROBUST STABILITY TEST FOR STRUCTURED LTV UNCERTAINTIES}

In this section, we present the main contributions of the paper, which basically generalizes the robust stability analysis method developed by Jönsson and Rantzer [1994] using the so-called swapping lemma (see Chou and Tits [1995] for a discretetime version).We first generalize the swapping lemma, which we then employ together with the bounding lemma to prove our main result. As a direct corollary, we finally provide a robust stability test in terms of an LMI feasibility problem.

\subsection{Generalized Swapping Lemma}

Lemma 7. (Swapping Lemma) Let $\Delta \in \mathcal{L}^{m \Delta \times n_{\Delta}}$ be a causal and bounded linear operator and let $H \in \mathcal{R H}_{\infty}^{m_{H} \times n_{H}}$ be a causal and stable transfer function that admits a realization of the form $H=\left[\begin{array}{c|c}A_{H} & B_{H} \\ \hline C_{H} & D_{H}\end{array}\right]$, where $A_{H} \in \mathbb{R}^{k_{H} \times k_{H}}, B_{H} \in$ $\mathbb{R}^{k_{H} \times n_{H}}, C_{H} \in \mathbb{R}^{m_{H} \times k_{H}}, D_{H} \in \mathbb{R}^{m_{H} \times n_{H}}$, with $A_{H}$ having all its eigenvalues strictly inside the unit disk. With $H_{B} \in \mathcal{R H}_{\infty}^{k_{H} \times n_{H}}$ and $H_{C} \in$ $\mathcal{R} \mathcal{H}_{\infty}^{m_{H} \times k_{H}}$ defined respectively as

$$
\begin{aligned}
& H_{B} \triangleq Z_{k_{H}}\left(Z_{k_{H}}-A_{H}\right)^{-1} B_{H}, \\
& H_{C} \triangleq C_{H}\left(Z_{k_{H}}-A_{H}\right)^{-1} Z_{k_{H}},
\end{aligned}
$$

and with $\mathcal{V}_{\Delta} \triangleq \Delta Z_{n_{\Delta}}^{-1}-Z_{m_{\Delta}}^{-1} \Delta$ denoting the variation of $\Delta$, we have

$$
\begin{aligned}
& \left(I_{m_{H}} \otimes \Delta\right)\left(H \otimes I_{n_{\Delta}}\right)-\left(H \otimes I_{m_{\Delta}}\right)\left(I_{n_{H}} \otimes \Delta\right) \\
& =\left(H_{C} \otimes I_{m_{\Delta}}\right)\left(I_{k_{H}} \otimes \mathcal{V}_{\Delta}\right)\left(H_{B} \otimes I_{n_{\Delta}}\right) .
\end{aligned}
$$

PROOF. Let us consider the identity

$$
\begin{gathered}
\quad\left[\left(I_{k_{H}}-Z_{k_{H}}^{-1} A_{H}\right) \otimes I_{m_{\Delta}}\right]\left[I_{k_{H}} \otimes\left(\Delta Z_{n_{\Delta}}^{-1}\right)\right] \\
=\left(I_{k_{H}} \otimes \mathcal{V}_{\Delta}\right) \\
+\left[I_{k_{H}} \otimes\left(Z_{m_{\Delta}}^{-1} \Delta\right)\right]\left[\left(I_{k_{H}}-Z_{k_{H}}^{-1} A_{H}\right) \otimes I_{n_{\Delta}}\right],
\end{gathered}
$$

which can be verified by adding/subtracting $\left[I_{k_{H}} \otimes\left(Z_{m_{\Delta}}^{-1} \Delta\right)\right]$ to/from the left hand side and rearranging the terms after employing the identity $\left(A_{H} \otimes I_{m_{\Delta}}\right)\left(I_{k_{H}} \otimes \Delta\right)=\left(I_{k_{H}} \otimes \Delta\right)\left(A_{H} \otimes I_{n_{\Delta}}\right)$. Moving the last term to the left hand side, left/right multiplying both sides of the equation, respectively, by $H_{C} \otimes I_{m_{\Delta}}$ and $H_{B} \otimes I_{n_{\Delta}}$, and finally employing the commutation identities $\left(I_{k_{H}} \otimes \Delta\right)\left(B_{H} \otimes I_{n_{\Delta}}\right)=\left(B_{H} \otimes I_{m_{\Delta}}\right)\left(I_{n_{H}} \otimes \Delta\right)$, $\left(C_{H} \otimes I_{m_{\Delta}}\right)\left(I_{k_{H}} \otimes \Delta\right)=\left(I_{m_{H}} \otimes \Delta\right)\left(C_{H} \otimes I_{n_{\Delta}}\right)$, we obtain

$$
\begin{gathered}
\left(I_{m_{H}} \otimes \Delta\right)\left(\left[C_{H}\left(Z_{k_{H}}-A_{H}\right)^{-1} B_{H}\right] \otimes I_{n_{\Delta}}\right) \\
-\left(\left[C_{H}\left(Z_{k_{H}}-A_{H}\right)^{-1} B_{H}\right] \otimes I_{m_{\Delta}}\right)\left(I_{n_{H}} \otimes \Delta\right) \\
\quad=\left(H_{C} \otimes I_{m_{\Delta}}\right)\left(I_{k_{H}} \otimes \mathcal{V}_{\Delta}\right)\left(H_{B} \otimes I_{n_{\Delta}}\right) .
\end{gathered}
$$

Adding/subtracting $\left(I_{m_{H}} \otimes \Delta\right)\left(D_{H} \otimes I_{n_{\Delta}}\right)=$ $\left(D_{H} \otimes I_{m_{\Delta}}\right)\left(I_{n_{H}} \otimes \Delta\right)$ to/from the left hand side, we end up with (10). 
Remark 8. The form of the swapping lemma for scalar repeated uncertainties, as it appears in Jönsson and Rantzer [1996]; Chou and Tits [1995], can be recovered from (10) as a special case corresponding to $m_{\Delta}=n_{\Delta}=1$. Another special case deserving interest is the case of full-block uncertainty considered with a repeated scalar LTI system, which is basically the case corresponding to $m_{H}=n_{H}=1$.

Remark 9. When a block-diagonal structured uncertainty, $\Delta=\left[I_{l_{i}} \otimes \Delta_{i}\right]_{i=1}^{\kappa} ; \Delta_{i} \in \mathcal{L}^{m_{i} \times n_{i}}$, is considered together with a transfer function that has a structure of the form $H=\left[H_{i} \otimes I_{m_{i}}\right]_{i=1}^{\kappa} ; H_{i} \in$ $\mathcal{R} \mathcal{H}_{\infty}^{q_{i} \times l_{i}}$, application of (10) for each block leads to

$$
\Delta_{\mathrm{l}} H_{\mathrm{r}}-H \Delta=H_{C} \tilde{\Delta} H_{B},
$$

where $H_{B}$ and $H_{C}$ are defined (consistently with (8) and (9)) as $H_{B}=\left[H_{i B} \otimes I_{n_{i}}\right]_{i=1}^{\kappa} ; H_{i B} \in$ $\mathcal{R} \mathcal{H}_{\infty}^{k_{i} \times l_{i}}, H_{C}=\left[H_{i C} \otimes I_{m_{i}}\right]_{i=1}^{\kappa} ; H_{i C} \in \mathcal{R} \mathcal{H}_{\infty}^{q_{i} \times k_{i}}$ respectively; $H_{\mathrm{r}}$ and $\Delta_{\mathrm{l}}$ are given by $H_{\mathrm{r}}=\left[H_{i} \otimes\right.$ $\left.I_{n_{i}}\right]_{i=1}^{\kappa}$ and $\Delta_{\mathrm{l}}=\left[I_{q_{i}} \otimes \Delta_{i}\right]_{i=1}^{\kappa} ;$ and $\tilde{\Delta}$ is an operator that is formed with the repeated forms of the variations of the sub-blocks of $\Delta$ according to $\tilde{\Delta}=\left[I_{k_{i}} \otimes \mathcal{V}_{\Delta_{i}}\right]_{i=1}^{\kappa}$. We would like to note that an implicit version of the swapping lemma (based on the commutation properties of the realization matrices of the LTI system with the LTV uncertainty) is given by Helmersson [1999].

\subsection{Main Result}

Theorem 10. Consider the setup of Problem 3. Assume that $(M, \tau \Delta)$ is well-posed for all $\Delta \in \boldsymbol{\Delta}_{\nu}^{s}$ and $\tau \in[0,1]$. Then, $M$ is robustly stable against $\boldsymbol{\Delta}_{\nu}^{s}$ if (5) is satisfied for some $\Pi \in \Pi_{\nu}^{s^{c}}$, with the multiplier set $\boldsymbol{\Pi}_{\nu}^{s^{c}}$ being defined as

$$
\Pi_{\nu}^{s^{c}} \triangleq\left\{\Pi_{R}+\Pi_{S}: \Pi_{R} \in \Pi_{R}, \Pi_{S} \in \Pi_{S}\right\},
$$

where

$$
\begin{aligned}
& \Pi_{R} \triangleq\left\{\begin{aligned}
\Pi_{R}= & {\left[\begin{array}{cc}
\Pi_{11} & 0 \\
0 & \Pi_{22}
\end{array}\right]: } \\
\Pi_{11}= & R^{*} X_{R 1} R+R_{B}^{*} V_{R} Y_{R} V_{R} R_{B} \\
\Pi_{22}= & R^{*} X_{R 2} X_{R 1}^{-1} X_{R 2} R-2 R^{*} X_{R 2} R \\
& +R^{*} X_{R 2} R_{C} V_{R} Y_{R}^{-1} V_{R} R_{C}^{*} X_{R 2} R \\
R= & {\left[R_{i} \otimes I_{m_{i}}\right]_{i=1}^{\kappa} \quad ; R_{i} \quad \in \mathcal{R H}_{\times}^{q_{i} \times l_{i}} } \\
X_{R 1}= & {\left[X_{R 1_{i}} \otimes I_{m_{i}}\right]_{i=1}^{\kappa} ; X_{R 1_{i}} \in \mathbb{P}^{q_{i} \times q_{i}} } \\
X_{R 2}= & {\left[X_{R 2_{i}} \otimes I_{m_{i}}\right]_{i=1}^{\kappa} ; X_{R 2_{i}} \in \mathbb{P}^{q_{i} \times q_{i}} } \\
Y_{R}= & {\left[Y_{R_{i}} \otimes I_{m_{i}}\right]_{i=1}^{\kappa} ; Y_{R_{i}} \in \mathbb{P}^{k_{i} \times k_{i}} }
\end{aligned}\right\}(13 \\
& \Pi_{S} \triangleq\left\{\begin{aligned}
\Pi_{S}= & {\left[\begin{array}{cc}
\Pi_{11} & \Pi_{12} \\
\Pi_{12}^{*} & 0
\end{array}\right]: } \\
\Pi_{11}= & E^{T} S_{B}^{*} V_{S} Y_{S} V_{S} S_{B} E \\
& +E^{T} X_{S} S_{C} V_{S} Y_{S}^{-1} V_{S} S_{C}^{*} X_{S}^{T} E \\
\Pi_{12}= & E^{T}\left(X_{S} S-S^{*} X_{S}^{T}\right) E \\
S= & {\left[S_{i} \otimes I_{m_{i}}\right]_{i=1}^{\kappa_{1}} ; S_{i} \in \mathcal{R} \mathcal{H}_{\infty}^{q_{i} \times l_{i}} } \\
X_{S}= & {\left[X_{S_{i}} \otimes I_{m_{i}}\right]_{i=1}^{\kappa_{1}} ; X_{S_{i}} \in \mathbb{R}^{l_{i} \times q_{i}} } \\
Y_{S}= & {\left[Y_{S_{i}} \otimes I_{m_{i}}\right]_{i=1}^{\kappa_{1}} ; Y_{S_{i}} \in \mathbb{P}^{k_{i} \times k_{i}} }
\end{aligned}\right\}
\end{aligned}
$$

Here; $R_{B}, R_{C}, S_{B}, S_{C}$ are defined for $R=\left[R_{i} \otimes\right.$ $\left.I_{m_{i}}\right]_{i=1}^{\kappa}$ and $S=\left[S_{i} \otimes I_{m_{i}}\right]_{i=1}^{\kappa_{1}}$ (consistently with (8) and (9)) as $R_{B}=\left[R_{i B} \otimes I_{m_{i}}\right]_{i=1}^{\kappa} ; R_{i B} \in$ $\mathcal{R H}_{\infty}^{k_{i} \times l_{i}}, R_{C}=\left[R_{i C} \otimes I_{m_{i}}\right]_{i=1}^{\kappa} ; R_{i C} \in \mathcal{R} \mathcal{H}_{\infty}^{q_{i} \times k_{i}}$, $S_{B}=\left[S_{i B} \otimes I_{m_{i}}\right]_{i=1}^{\kappa_{1}} ; \quad S_{i B} \in \mathcal{R H}_{\infty}^{k_{i} \times l_{i}}, S_{C}=$ $\left[S_{i C} \otimes I_{m_{i}}\right]_{i=1}^{\kappa_{1}} ; \quad S_{i C} \in \mathcal{R} \mathcal{H}_{\infty}^{q_{i} \times k_{i}} ; V_{R}$ and $V_{S}$ are matrices that contain the information on rateof-variation bounds, with their definitions being $V_{R} \triangleq\left[\sqrt{\nu_{i}} I_{k_{i}} \otimes I_{m_{i}}\right]_{i=1}^{\kappa} \& V_{S} \triangleq\left[\sqrt{\nu_{i}} I_{k_{i}} \otimes\right.$ $\left.I_{m_{i}}\right]_{i=1}^{\kappa_{1}}$; and $E$ is a matrix that is introduced to assure dimensional compatibility by extending the relevant operators with zeros according to $E \triangleq\left[\begin{array}{ll}I_{\eta_{1}} & 0_{\eta_{1} \times \eta_{2}}\end{array}\right]$ with $\eta_{1}=\sum_{i=1}^{\kappa_{1}} l_{i} m_{i}$ and $\eta_{2}=$ $\sum_{i=\kappa_{1}+1}^{\kappa} l_{i} m_{i}=\eta-\eta_{1}$.

PROOF. The proof is based on an application of the IQC stability theorem and follows somewhat parallel lines to the work of Jönsson and Rantzer [1996]. We prove the theorem by showing that, for all $\Delta \in \boldsymbol{\Delta}_{\nu}^{s}$ and all $\tau \in[0,1]$, we have $\tau \Delta \in \boldsymbol{\Delta}_{\Pi}$ for any $\Pi \in \boldsymbol{\Pi}_{R}$ as well as for any $\Pi \in \boldsymbol{\Pi}_{S}$. It then follows that, for all $\tau \in[0,1]$, we have $\tau \Delta \in \boldsymbol{\Delta}_{\Pi}$ for any $\Pi \in \Pi_{\nu}^{s^{c}}$, which means according to the IQC stability theorem that the feedback system is robustly stable, provided that the frequency domain inequality of (5) is satisfied for some $\Pi \in \Pi_{\nu}^{s^{c}}$.

We can show that, $\Delta \in \boldsymbol{\Delta}_{\nu}^{s}$ implies $\Delta \in \boldsymbol{\Delta}_{\Pi}$ for any $\Pi \in \boldsymbol{\Pi}_{R}$, by considering the self-adjoint operator $2(H \Delta)^{*}(H \Delta)$ for some $H=U R$, where $R=\left[R_{i} \otimes I_{m_{i}}\right]_{i=1}^{\kappa} ; \quad R_{i} \in \mathcal{R H}_{\infty}^{q_{i} \times l_{i}} ; U=\left[U_{i} \otimes\right.$ $\left.I_{m_{i}}\right]_{i=1}^{\kappa} ; U_{i}, U_{i}^{-1} \in \mathbb{R}^{q_{i} \times q_{i}}$ and applying the general form of the swapping lemma given in (11) for structured uncertainties, together with the bounding lemma to write

$$
\begin{gathered}
2 \Delta^{*} H^{*} H \Delta \leq H^{*} \Delta_{l}^{*} X \Delta_{l} H+\Delta^{*} H^{*} X^{-1} H \Delta \\
+H_{B}^{*} \tilde{\Delta}^{*} Y \tilde{\Delta} H_{B}+\Delta^{*} H^{*} H_{C} Y^{-1} H_{C}^{*} H \Delta,
\end{gathered}
$$

where we restrict the choices of $X$ and $Y$ to the structured matrices of the form $X=K^{T} K=$ $\left[K_{i}^{T} K_{i} \otimes I_{m_{i}}\right]_{i=1}^{\kappa} ; K_{i}, K_{i}^{-1} \in \mathbb{R}^{q_{i} \times q_{i}}$ and $Y=$ $L^{T} L=\left[L_{i}^{T} L_{i} \otimes I_{m_{i}}\right]_{i=1}^{\kappa} ; \quad L_{i}, L_{i}^{-1} \in \mathbb{R}^{k_{i} \times k_{i}}$. Thanks to these specific choices of the structures of $X$ and $Y$, it can easily be shown (by using the Kronecker product commutation identity and by employing $\left\|\Delta_{i}\right\|_{\mathrm{i} 2} \leq 1$ and $\left.\left\|\mathcal{V}_{\Delta_{i}}\right\|_{\mathrm{i} 2} \leq \nu_{i}\right)$ that $H^{*} \Delta_{1}^{*} X \Delta_{1} H \leq H^{*} X H$ and $H_{B}^{*} \tilde{\Delta}^{*} Y \tilde{\Delta} H_{B} \leq H_{B}^{*} V_{R}^{2} Y V_{R}^{2} H_{B}$. Inserting $H=$ $U R ; H_{B}=R_{B} ; H_{C}=U R_{C}$, from which it follows that $H^{*} H=R^{*} U^{T} U R=R^{*} X_{R 2} R ; H^{*} X H=$ $R^{*} X_{R 1} R ; H^{*} H_{C}=R^{*} X_{R 2} R_{C} ; H^{*} X^{-1} H=$ $R^{*} X_{R 2} X_{R 1}^{-1} X_{R 2} R$, with the definitions $X_{R 1} \triangleq$ $U^{T} X U ; X_{R 2} \triangleq U^{T} U$, and setting $Y=V_{R}^{-1} Y_{R} V_{R}^{-1}$ for purposes of annihilating in the channels with $\nu_{i}=0$, the terms attributed to the swapping affect (which is clearly nonexistent for the time-invariant channels), we conclude that $\Delta \in \boldsymbol{\Delta}_{\Pi}$. Since we have $\Pi_{12}=0$ for $\Pi \in \Pi_{R}$, we conclude that $\tau \Delta \in \boldsymbol{\Delta}_{\Pi}$ for all $\Pi \in \boldsymbol{\Pi}_{R}$ and for all $\tau \in[0,1]$. 
The fact that $\Delta \in \boldsymbol{\Delta}_{\nu}^{s}$ implies $\Delta \in \boldsymbol{\Delta}_{\Pi}$ for any $\Pi \in \Pi_{S}$ can be proved by first considering the self-adjoint part of the uncertainty $\left(\Delta=\left[I_{l_{i}} \otimes\right.\right.$ $\left.\Delta_{i}\right]_{i=1}^{\kappa_{1}}$ ) together with some $H=X_{S} S$, where $S=\left[S_{i} \otimes I_{m_{i}}\right]_{i=1}^{\kappa_{1}} ; S_{i} \in \mathcal{R} \mathcal{H}_{\infty}^{q_{i} \times l_{i}} ; X_{S}=\left[X_{i} \otimes\right.$ $\left.I_{m_{i}}\right]_{i=1}^{\kappa_{1}} ; \quad X_{i} \in \mathbb{R}^{l_{i} \times q_{i}}$, and again employing the swapping and the bounding lemmas to write

$$
\begin{aligned}
\Delta^{*}\left(H-H^{*}\right) & +\left(H^{*}-H\right) \Delta \\
\leq & H_{B}^{*} \tilde{\Delta}^{*} Y \tilde{\Delta} H_{B}+H_{C} Y^{-1} H_{C}^{*},
\end{aligned}
$$

where $Y$ is assumed to be of the form $Y=$ $\left[L_{i}^{T} L_{i} \otimes I_{m_{i}}\right]_{i=1}^{\kappa_{1}} ; \quad L_{i}, L_{i}^{-1} \in \mathbb{R}^{k_{i} \times k_{i}}$. Inserting $H_{B}^{*} \tilde{\Delta}^{*} Y \tilde{\Delta} H_{B} \leq S_{B}^{*} V_{S}^{2} Y V_{S}^{2} S_{B} ; H=X_{S} S ; H_{B}=$ $S_{B} ; H_{C}=X_{S} S_{C}$, setting $Y=V_{S}^{-1} Y_{S} V_{S}^{-1}$ due to the concerns for the time-invariant channels, and finally introducing the extension matrix $E=$ $\left[\begin{array}{ll}I_{\eta_{1}} & 0_{\eta_{1} \times \eta_{2}}\end{array}\right]$ to rewrite the inequality for the complete $\Delta$ operator, we conclude that $\Delta \in \boldsymbol{\Delta}_{\Pi}$. Since $\Pi_{11} \geq 0$ and $\Pi_{22}=0$ for $\Pi \in \Pi_{S}$, we have $\tau \Delta \in \boldsymbol{\Delta}_{\Pi}$ for all $\Pi \in \boldsymbol{\Pi}_{S}$ and $\tau \in[0,1]$.

Remark 11. The multiplier sets defined in (13) and (14) have some differences than those in Jönsson and Rantzer [1994, 1996]. In the multiplier sets defined by Jönsson and Rantzer [1994], we have $X_{R 1}=X_{R 2}$ and also the matrices $Y_{R}$ and $Y_{S}$ are restricted to be positive-definite matrices of scalar repeated sub-blocks. In Jönsson and Rantzer [1996], $Y_{R}$ and $Y_{S}$ are both set to identity matrices of relevant sizes. On the other hand, $Y_{R}$ and $Y_{S}$ as defined in (13) and (14) can have full sub-blocks at the channels where the uncertainty has repeated scalar sub-blocks (i.e. for $m_{i}=1$ ). This (as well as not necessarily equal $X_{R 1}$ and $X_{R 2}$ ) introduces extra freedom in the search for a suitable multiplier that satisfies (5). Moreover, it will become clear in the next subsection that an efficient search is possible over $Y_{R}$ and $Y_{S}$, which is also not discussed by Jönsson and Rantzer [1994]. In the LTI case, $V_{R}=0 ; V_{S}=0$, we recover the well-known multiplier class corresponding to the D-G scaling with $X_{R 1}=X_{R 2}$, which can be imposed in this case without introducing any conservatism. However, due to the -somewhatdifferent nature of the discrete-time setting, it is not straightforward to conclude that the multipliers should be constant for arbitrarily fast timevarying uncertainties, i.e. for $V_{R}=\sqrt{2} I, V_{S}=\sqrt{2} I$.

\subsection{LMI Test for Robust Stability Analysis}

We can apply the Schur complement lemma to linearize inequality (5) in the matrix variables (by a suitable extension of the plant and the multiplier, as also done by Jönsson and Rantzer [1996]), and then employ the KYP lemma (after factorizing the extended multiplier to separate the matrix variables from the dynamic part) to end up with the following stability test:
Corollary 12. Consider the setup of Problem 3 and assume that $(M, \tau \Delta)$ is well-posed for all $\Delta \in$ $\boldsymbol{\Delta}_{\nu}^{s}$ and $\tau \in[0,1]$. With $R=\left[R_{i} \otimes I_{m_{i}}\right]_{i=1}^{\kappa} ; R_{i} \in$ $\mathcal{R} \mathcal{H}_{\infty}^{q_{i} \times l_{i}}$ and $S=\left[S_{i} \otimes I_{m_{i}}\right]_{i=1}^{\kappa_{1}} ; \quad S_{i} \in \mathcal{R H}_{\infty}^{q_{i} \times l_{i}}$ being given transfer functions (e.g. with $R_{i}=$ $S_{i}=\left[I_{l_{i}} \ldots Z_{l_{i}}^{-p_{i}}\right]^{T}$ and thus $q_{i}=\left(p_{i}+1\right) l_{i}$, $\left.k_{i}=p_{i} l_{i}\right)$, define $\Phi$ as

$$
\Phi \triangleq\left[\begin{array}{cccc}
R M & 0 & 0 & 0 \\
R & 0 & 0 & 0 \\
R & 0 & I & R_{C} V_{R} \\
V_{R} R_{B} M & 0 & 0 & 0 \\
-E & 0 & 0 & 0 \\
S E M & 0 & 0 & 0 \\
E M & 0 & 0 & 0 \\
S E & S_{C} V_{S} & 0 & 0 \\
V_{S} S_{B} E M & 0 & 0 & 0 \\
0 & I & 0 & 0 \\
0 & 0 & I & 0 \\
0 & 0 & 0 & I
\end{array}\right] .
$$

Then, $M$ is robustly stable against $\boldsymbol{\Delta}_{\nu}^{s}$ if there exist: (i) a symmetric matrix $P=P^{T} \in \mathbb{R}^{k_{\Phi} \times k_{\Phi}}$; (ii) a structured matrix $Q \in \mathbb{Q}$; and (iii) an $\epsilon \in \mathbb{R}_{+}$ such that (7) is satisfied, with $\left[\begin{array}{l|l}A_{\Phi} & B_{\Phi} \\ \hline C_{\Phi} & D_{\Phi}\end{array}\right]$ denoting a minimal realization of $\Phi$ and $\mathbb{Q}$ being defined as

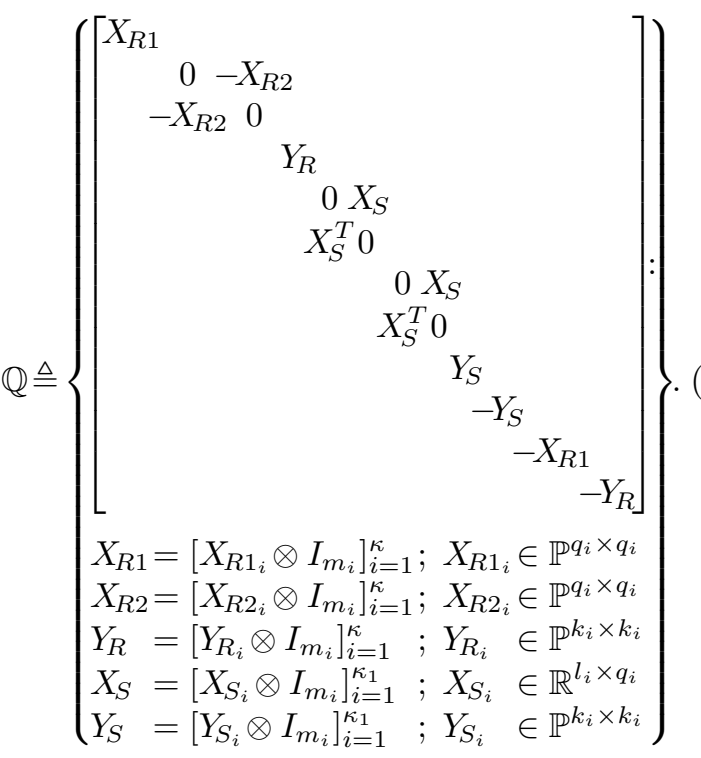

Remark 13. The method identified by Corollary 12 provides to Problem 3 a solution that is numerically efficient for problems of moderate size, also allowing gradual reduction of the conservatism (for instance by larger $p_{i}$ ). With $\nu_{i}=\nu ; \forall i$, we obtain the setup considered by Poolla and Tikku [1995], who provide a conservative estimate of the maximum allowable rate-of-variation bound for robust stability. The form of this estimate does not allow an efficient search. On the other hand, we can employ the test of Corollary 12 (with $V_{R}=\nu I$ and $V_{S}=\nu I$ ) in a bisection type search to determine an estimate of the maximum allowable rate-of-variation for the uncertainties that are not jeopardizing stability. 


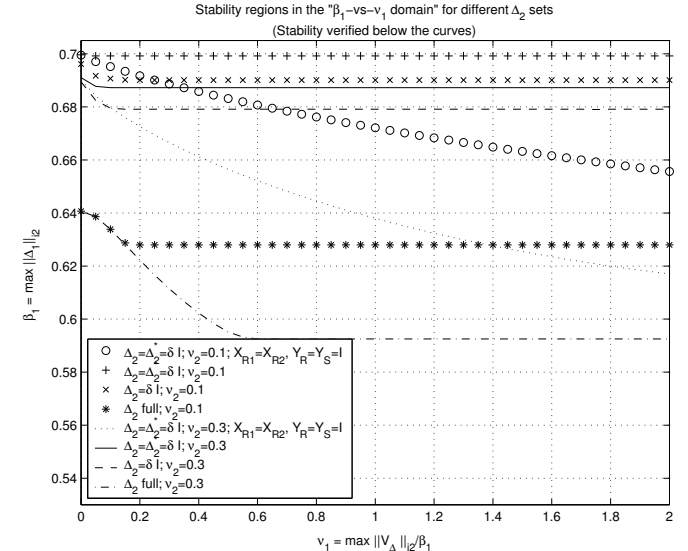

Fig. 2. Robust stability regions in $\nu_{1}-\beta_{1}$ domain.

\section{ILLUSTRATIVE EXAMPLE}

We provide in this section some analysis results (obtained via an implementation of the test described by Corollary 12 in MATLAB-LMILAB within a bisection search) for a strictly proper plant $M$ with the realization

$$
\left[\begin{array}{c|c}
A_{M} & B_{M} \\
\hline C_{M} & D_{M}
\end{array}\right]=\left[\begin{array}{rrr|rrr}
0.04 & 0.09 & -0.14 & 0.02 & 0.22 & 0.06 \\
0.09 & 0.25 & 0.05 & -0.24 & -0.08 & 0.64 \\
-0.04 & -0.06 & -0.04 & 0.22 & -0.82 & 0.40 \\
\hline 1.00 & -1.00 & 2.00 & 0.00 & 0.00 & 0.00 \\
-1.00 & 2.00 & -1.00 & 0.00 & 0.00 & 0.00 \\
1.00 & 1.00 & 1.00 & 0.00 & 0.00 & 0.00
\end{array}\right],
$$

and block-diagonal structured LTV uncertainties with two uncertainty channels, $\Delta_{1} \in \mathcal{L}^{1 \times 1}$ and $\Delta_{2} \in \mathcal{L}^{2 \times 2}$. With magnitude and normalized rateof-variation bounds imposed on $\Delta_{i}$ denoted by $\beta_{i}=\max \left\|\Delta_{i}\right\|_{\mathrm{i} 2}$ and $\nu_{i}=\max \left\|\mathcal{V}_{\Delta_{i}}\right\|_{\mathrm{i} 2} / \beta_{i}$, we present in Figure 2 the robust stability regions (verified using bases of the form $R_{i}=S_{i}=$ $\left[I Z^{-1}\right]^{T}$ ) in the $\beta_{1}-\nu_{1}$ domain (as $\nu_{1}$ varies from 0 to 2 ), for three different structure (self-adjoint scalar repeated, arbitrary scalar repeated and full blocks) and two different normalized maximal rate-of-variation $\left(\nu_{2}=0.1\right.$ and $\left.\nu_{2}=0.3\right)$ choices of $\Delta_{2}$, for all of which $\beta_{2}=0.45$. As expected, the region where the stability is verified (usually) gets larger as the restrictions on the structure and the nature of the second block are relaxed. Moreover, the verified region gets significantly smaller (especially for large $\nu_{1}$ values) for $X_{R 1}=$ $X_{R 2}, Y_{R}=I$ and $Y_{S}=I$, with which we get the multipliers of Jönsson and Rantzer [1996].

\section{CONCLUDING REMARKS}

We have generalized the stability analysis method developed by Jönsson and Rantzer [1994, 1996] for structured LTV uncertainties with repeated scalar sub-blocks to general structured LTV uncertainties. The results admit generalizations to the case of non-square plants/uncertainties and to continuous-time setting with modest difficulty. A naturally challenging research direction is the investigation of the degree of conservatism involved in the method and in particular the possibility of (or conditions for) exactness.

\section{ACKNOWLEDGEMENT}

Supported by the Technology Foundation STW, applied science division of NWO and the technology programme of the Ministry of Economic Affairs.

\section{REFERENCES}

Y.-S Chou and A. L. Tits. On robust stability under slowly-varying memoryless uncertainty. In Proc. 34th IEEE Conf. Dec. ES Cont., pp.43214326, New Orleans, Los Angeles, Dec. 1995.

A. Helmersson. An IQC-based stability criterion for systems with slowly varying parameters. In Proc. 14th IFAC World Cong., pp.525-530, Beijing, China, July 1999.

U. Jönsson. Lecture notes on integral quadratic constraints. Div. of Optimization and Systems Theory, Dept. of Mathematics, Royal Inst. of Tech., Stockholm, Sweden, May 2001.

U. Jönsson and A. Rantzer. Systems with uncertain parameters - time-variations with bounded derivatives. In Proc. 33rd IEEE Conf. Dec. \& Cont., pp.3074-3079, Lake Buena Vista, FL, Dec. 1994.

U. Jönsson and A. Rantzer. Systems with uncertain parameters - time-variations with bounded derivatives. Int. Journ. Rob. \&5 Nonl. Cont., 6 (9-10):969-982, Nov. 1996.

A. Megretski and A. Rantzer. System analysis via integral quadratic constraints. IEEE Trans. Aut. Cont., 42(6):819-830, June 1997.

G. Meinsma, T. Iwasaki, and M. Fu. When is $(D, G)$-scaling both necessary and sufficient. IEEE Trans. Aut. Cont., 45(9):17551759, Sept. 2000.

A. Packard and J. C. Doyle. The complex structured singular value. Automatica, 29(1):71-109, Jan. 1993.

F. Paganini and J. C. Doyle. Analysis of implicitly defined systems. In Proc. 33rd IEEE Conf. Dec. 83 Cont., pp.3673-3678, Lake Buena Vista, FL, Dec. 1994.

K. Poolla and A. Tikku. Robust performance against time-varying structured perturbations. IEEE Trans. Aut. Cont., 40(9):15891602, Sept. 1995.

A. Rantzer. On the Kalman-Yakubovich-Popov lemma. Syst. $\& 3$ Cont. Lett., 28(1):7-10, June 1996.

J. S. Shamma. Robust stability with timevarying structured uncertainty. IEEE Trans. Aut. Cont., 39(4):714-724, April 1994.

A. Tchernychev and A. Sideris. Robust discretetime control design with time-varying parametric uncertainties. Int. Journ. Rob. \& Nonl. Cont., 9(4):199-213, April 1999.

A. Tchernychev and A. Sideris. Multiplier-based robust $\mathcal{H}_{\infty}$ design with time-varying uncertainties. Automatica, 36(4):579-586, April 2000.

J. C. Willems. The Analysis of Feedback Systems. The MIT Press, MA, 1971. 